\title{
Tricaprin Rescues Myocardial Abnormality in a Mouse Model of Triglyceride Deposit Cardiomyovasculopathy
}

\author{
Akira Suzuki $^{1 *}$, Satoshi Yamaguchi ${ }^{1}$, Ming Li ${ }^{1}$, Yasuhiro Hara ${ }^{1}$, Hideyuki Miyauchi ${ }^{2}$, \\ Yoshihiko Ikeda ${ }^{3}$, Bo Zhang ${ }^{4}$, Masahiro Higashi ${ }^{5}$, Yasuyuki Ikeda ${ }^{1}$, Atsuko Takagi ${ }^{1}$, \\ Hironori Nagasaka ${ }^{6}$, Kunihisa Kobayashi ${ }^{7}$, Yasuhiro Magata ${ }^{8}$, Toshiaki Aoyama ${ }^{9}$, and \\ Ken-ichi Hirano ${ }^{1}$ \\ ${ }^{1}$ Laboratory of Cardiovascular Disease, Novel, Non-Invasive, and Nutritional Therapeutics (CNT), Graduate School of Medicine, Osaka \\ University, 6-2-3 Furuedai, Suita, Osaka 565-0874, JAPAN \\ ${ }^{2}$ Department of Cardiovascular Medicine, Chiba University Graduate School of Medicine, 1-8-1 Inohana, Chuo-ku, Chiba 260-8670, JAPAN \\ ${ }^{3}$ Department of Pathology, National Cerebral and Cardiovascular Center, 5-7-1 Fujishirodai, Suita, Osaka 565-8565, JAPAN \\ ${ }^{4}$ Department of Biochemistry, Fukuoka University Medical School, 8-19-1 Nanakuma, Jonan-ku, Fukuoka 814-0180, JAPAN \\ ${ }^{5}$ Department of Radiology, Osaka National Hospital, 2-1-14 Hoenzaka, Chuo-ku, Osaka 540-0006, JAPAN \\ ${ }^{6}$ Department of Pediatrics, Takarazuka City Hospital, 4-5-1 Obama, Takarazuka, Hyogo 665-0827, JAPAN \\ ${ }^{7}$ Department of Endocrinology and Diabetes Mellitus, Fukuoka University Chikushi Hospital, 1-1-1 Zokumyoin, Chikushino, Fukuoka 818-8502, \\ JAPAN \\ ${ }^{8}$ Preeminent Medical Photonics Education \& Research Center, Hamamatsu University School of Medicine, 1-20-1 Handayama, Higashi-ku, \\ Hamamatsu, Shizuoka 431-3192, JAPAN \\ ${ }^{9}$ Daitocacao Co.,LTD. Annex 2-3-23 Shimomeguro, Meguro-ku,Tokyo, 153-0064 JAPAN
}

\begin{abstract}
Triglyceride deposit cardiomyovasculopathy (TGCV) is an intractable cardiovascular disease for which a specific treatment is urgently required. In TGCV, adipose triglyceride lipase (ATGL) deficiency results in the abnormal intracellular metabolism of long-chain fatty acid (LCFA) which leads to TG deposition. Medium-chain triglycerides have been used as an important functional food for various human diseases. To address the potential activities of tricaprin, a medium-chain triglyceride, on cardiac dysfunctions of TGCV, we examined the effects of tricaprin diet on Atgl knock out (KO) mice, an animal model for TGCV. Cardiac imaging tests showed that the tricaprin diet reduced TG accumulation, resulting from improvement of LCFA metabolism, and improved left ventricular function in Atgl KO mice compared to that in mice fed the control diet. In conclusion, tricaprin improved myocardial abnormality in the TGCV model, thus, it may be useful for the treatment of patients with TGCV.
\end{abstract}

Key words: adipose triglyceride lipase, capric acid, iodine-123- $\beta$-methyl iodophenyl-pentadecanoic acid, medium-chain triglyceride, tricaprin, triglyceride deposit cardiomyovasculopathy

\section{Introduction}

Triglyceride deposit cardiomyovasculopathy (TGCV) is a rare and intractable cardiovascular disease that was first reported among patients requiring cardiac transplantation in Japan ${ }^{1)}$. TGCV is characterized by a massive accumulation of triglyceride (TG) in the myocardium ${ }^{2}$ and coronary arteries $^{3)}$ which leads to severe congestive heart failure and angina pectoris. TGCV is classified into primary and idiopathic types. Primary TGCV is caused by genetic mutations
Abbreviations: ATGL, adipose triglyceride lipase; BMIPP, iodine-123- $\beta$-methyl iodophenyl-pentadecanoic acid; CT, computed tomography; FA, fatty acid; HE, hematoxylin and eosin; KO, knockout; LCFA, long-chain fatty acid; LVEF, left ventricular ejection fraction; MCFA, medium chain fatty acid; ROI, region of interest; SPECT, single-photon emission computed tomography; TG, triglyceride; TGCV, triglyceride deposit cardiomyovasculopathy; WOR, washout rate; WT, wild type

\footnotetext{
*Correspondence to: Akira Suzuki, Laboratory of Cardiovascular Disease, Novel, Non-Invasive, and Nutritional Therapeutics (CNT), Graduate School of Medicine, Osaka University, 6-2-3 Furuedai, Suita, Osaka 565-0874, JAPAN

E-mail: suzukia@cnt-osaka.com

Accepted April 5, 2018 (received for review March 7, 2018)

Journal of Oleo Science ISSN 1345-8957 print / ISSN 1347-3352 online

http://www.jstage.jst.go.jp/browse/jos/ http://mc.manusriptcentral.com/jjocs
} 
in PNPLA2 which encodes adipose triglyceride lipase (ATGL), a rate-limiting enzyme in the hydrolysis of intracellular TG and the release of free long-chain fatty acids $(\mathrm{LCFAs})^{4,5)}$. Idiopathic TGCV does not carry PNPLA2 mutations ${ }^{6)}$, but is associated with a marked reduction in ATGL activity in peripheral leukocytes ${ }^{7)}$.

Clinically, TGCV with ATGL deficiency causes severe heart failure with a poor prognosis ${ }^{1,8,9)}$. Hence, the development of specific treatment for TGCV is an urgent requirement. Our previous in vitro experiments on fibroblasts derived from patients with TGCV showed that LCFAs, the major energy source for the normal heart, accumulate as TG in cytoplasmic lipid droplets owing to ATGL deficiency $^{10,11}$. To resolve the above-mentioned toxicity of LCFAs, we explored the development of nutritional therapeutic agents from medium-chain fatty acids (MCFAs) for the following reasons: 1) MCFAs have a long and safe history as they have been used clinically for 50 years in patients with metabolic and neurological disorders ${ }^{12-14} ; 2$ ) MCFAs are an alternative energy source to LCFA and glucose $\mathrm{e}^{15,16)}$; and 3) the intracellular metabolism of MCFAs was shown to be different from that of LCFAs ${ }^{17)}$. It was shown that MCFAs activated FA oxidation enzymes in the rat liver when oils containing medium-chain triglycerides $(\mathrm{MCTs})$ were administered orally to rats ${ }^{18)}$. Moreover, the effects of MCTs on body fat have been evaluated and it was suggested that an MCT diet reduced body weight in humans ${ }^{19,20)}$.

Previous studies have shown that TG deposition in cardiomyocytes in Atgl KO mice resulted in cardiac phenotypes that were similar to those observed in patients with $\mathrm{TGCV}^{5,10)}$. In the present study, the potential beneficial effects of tricaprin, the TG form of capric acid (a type of MCFA) on the cardiac TG deposition and dysfunctions were examined in Atgl KO mice.

\section{Experimental procedure}

\subsection{Tricaprin}

Purified tricaprin (99\%) was obtained from Yashiro Co. Ltd (Osaka, Japan). Our recent study confirmed the safety of tricaprin in canine toxicity tests ${ }^{21)}$.

\subsection{Mouse model}

Atgl KO mice were kindly provided by Professor Rudolf Zechner (University of Graz, Austria) ${ }^{5}$. Four-week-old Atgl KO mice were divided into tricaprin $(+)$ and tricaprin $(-)$ diet groups. In the tricaprin $(+)$ diet, $8 \%$ of the natural fat was replaced with tricaprin (Yashiro, Osaka, Japan) whereas the tricaprin $(-)$ diet contained $10 \%$ natural fat. The experimental protocol was approved by the Ethics Review Committee for Animal Experimentation of Osaka and Hamamatsu University School of Medicine. All experiments were performed in accordance with the guidelines for animal use approved by the Institutional Animal Care and Use Committee of Osaka University Graduate School of Medicine (approval number: 27-070-001) and Hamamatsu University School of Medicine (approval number: 2012010). Wild-type (WT) C57Bl/6 mice fed the tricaprin (-) diet were used as the control.

\subsection{Animal experiments}

All analyses described in this section were performed on 7-week-old Atgl KO mice, because the KO mice over 8 weeks of age could not tolerate anesthesia owing to heart failure.

2.3.1 Myocardial iodine-123- $\beta$ methyl iodophenyl-pentadecanoic acid (BMIPP) scintigraphy by single-photon emission computed tomography (BMIPP-SPECT)

LCFA clearance at the cellular level can be detected by in vivo myocardial scintigraphy using BMIPP, a radiolabeled analog of LCFA ${ }^{22-24)}$. We recently reported that patients with TGCV showed a defective washout of BMIPP in a scintigraphic analysis ${ }^{2}$. To evaluate LCFA metabolism in the heart of Atgl KO mice in this study, BMIPP-SPECT was used. The Atgl KO mice fed the tricaprin (+) or tricaprin $(-) \operatorname{diet}(n=4$ in each group) were evaluated by using myocardial BMIPP-SPECT. BMIPP was obtained from Nihon Medi-Physics Co. Ltd., Tokyo, Japan, and injected into the jugular vein of mice under isoflurane anesthesia. BMIPP was then traced by SPECT/CT(Flex X-SPECT/CT, Gamma Medica-Ideas Inc., CA, USA). The SPECT images were obtained at $1 \mathrm{~h}$ and $4 \mathrm{~h}$ after the injection of BMIPP. The regions of interest(ROIs) were placed on the myocardium-based on CT images, and the decay-corrected radioactivity uptake in each ROI was obtained. Washout rates (WORs), used to evaluate LCFA metabolism, were calculated from the formula[(BMIPP uptake $(1 \mathrm{~h})$-remaining $\operatorname{BMIPP}(4 \mathrm{~h})) /$ BMIPP uptake (1 h) ].

2.3.2 Measurements of myocardial lipid deposition and left ventricular ejection fraction (LVEF) by CT

Micro-CT has been used to measure cardiac function in rodents $^{25,26)}$. In the present experiment, micro-CT (Latheta LCT-200, Hitachi-Aloka Medical, Tokyo, Japan) was used to measure cardiac lipid deposition and function in the mouse heart. The myocardial lipid deposition was examined in KO mice fed the tricaprin $(+)$ or $\operatorname{tricaprin}(-) \operatorname{diet}(\mathrm{n}=5$ in each group). The CT values (in Hounsfield units, HU) were measured in four regions (the septum and the anterior, posterior, and lateral walls of the heart) and the mean CT value was calculated from the CT values of four points.

The KO mice that were fed the tricaprin $(+)$ or tricaprin $(-) \operatorname{diet}(\mathrm{n}=7$ in each group) were examined by using a previously reported method for measurement of $\mathrm{LVEF}^{25)}$. After the mice were anesthetized, $30 \mu \mathrm{L}$ of contrast agent (ExiTron nano 12000, Miltenyi Biotec, Germany) was injected into the subclavian vein of the mice. The systolic 
and diastolic heart volumes were examined by using microCT. Images were obtained after every $96 \mu \mathrm{m}$, which separated the LV into segments; the volume of each segment was calculated by multiplication of the area by 96 . The total LV volume was calculated from addition of all segmental volumes. LVEF was determined after the systolic and diastolic volumes of the whole heart were calculated. $\mathrm{EF}$ was calculated from the following formula:

$$
\mathrm{EF}=\frac{\mathrm{LVEDV}-\mathrm{LVESV}}{\mathrm{LVEDV}} \times 100(\%)
$$

where LVEDV is the LV end-diastolic volume, and LVESV is the LV end-systolic volume.

\subsubsection{Histological analysis}

The mice hearts were fixed in formalin. The paraffin block sections were stained with hematoxylin and eosin (HE) and Masson's trichrome, and examined by using a microscope (Olympus, Tokyo, Japan).

\subsection{Statistical analysis}

The data are presented as the mean \pm standard error. A $p$ value of $<0.05$ was considered to indicate statistical significance.

\section{Results}

\subsection{Tricaprin diet improved LCFA WOR in the heart of Atgl KO mice}

Similar to that observed in patients with $\mathrm{TGCV}^{2)}$, the WORs of BMIPP were considerably reduced in the KO mice fed the tricaprin ( - ) $\operatorname{diet}($ Fig. 1A and 1B), In contrast, the WORs of BMIPP in KO mice fed the tricaprin $(+)$ diet were markedly higher than those in mice fed the tricaprin $(-)$ $\operatorname{diet}($ Fig. $1 \mathrm{~A}$ and $1 \mathrm{~B}$ ). As BMIPP is known to be incorporated into the cytoplasmic TG pool and oxidized by the cardiac mitochondria of rodents, similar to native LCFA ${ }^{27)}$, the increased WOR indicated that the tricaprin $(+)$ diet might facilitate LCFA metabolism in the heart of the KO mice.

\subsection{Tricaprin diet ameliorated lipid accumulation in the heart of $A t g / \mathrm{KO}$ mice}

The myocardial CT values shown in Fig. 2A and 2B reflective of the tissue TG content in patients with $\mathrm{TGCV}^{6,9)}$. The CT value in the WT group was $125 \pm 9.6 \mathrm{HU}$. In contrast, the CT values of the KO mice fed the tricaprin $(-)$ diet were low. The tricaprin $(+)$ diet-fed group showed significantly higher CT values than the tricaprin $(-)$ diet-fed group $\left(-27.5 \pm 5.7 \mathrm{HU}\right.$ vs. $8.1 \pm 5.5 \mathrm{HU},{ }^{*} p<0.01$ ) (Fig. 2B). This suggested that the tricaprin $(+)$ diet reduced fat accumulation in the heart of $A \operatorname{tgl} \mathrm{KO}$ mice.

Histological analysis was performed to confirm the observations obtained by using micro-CT. In 7-week-old KO mice, massive vacuoles were present in cardiomyocyte and fibrotic changes were observed after Masson's trichrome staining (Fig. 2C), as shown in previous results ${ }^{5}$. These features were attenuated in $\mathrm{KO}$ mice fed the tricaprin $(+)$ $\operatorname{diet}$ (c and $f$ in Fig. 2C).

\subsection{Tricaprin diet improved LV function in the heart of Atgl $\mathrm{KO}$ mice}

We assessed the effect of the tricaprin $(+)$ diet on cardiac function(Fig. 3). The LVEF in the KO mice fed the tricaprin $(+)$ diet was significantly higher than that in the KO mice fed the tricaprin ( -$) \operatorname{diet}(30 \pm 12 \%$ vs. $15 \pm 9 \%, p$ $<0.01)$. LVEF in the KO mice fed the tricaprin $(+)$ diet was comparable with that of WT mice fed tricaprin $(-)$ diet, which indicated that $\mathrm{LV}$ function was restored in the KO mice fed the tricaprin $(+)$ diet.

\section{Discussion}

There are several hypotheses that may explain the beneficial effects of the tricaprin (+) diet in ATGL-deficient conditions. First, capric acid can be used and oxidized as an alternative energy source, as reported in patients with other rare genetic disorders, such as very long-chain acylCoA dehydrogenase and malonyl-CoA decarboxylase deficiencies; in contrast, very long chain fatty acils and LCFAs, respectively, cannot be metabolized owing to defects in the corresponding enzymes ${ }^{28,29)}$. Second, capric acid might facilitate TG hydrolysis via certain MCFA-specific pathways at the cellular level, as previously suggested by a detailed biochemical analysis ${ }^{17)}$. Third, the tricaprin $(+)$ diet contains relatively low (2\%) natural fat, and it is potentially, not tricaprin, but the low natural fat content, that contributes to the present observations; however, this is unlikely, because a low-fat diet paradoxically induced fat accumula$\operatorname{tion}^{30)}$ and shortened the life span of Atgl KO mice (data not shown).

Capric acid has been reported to increase the capacity for fatty acid oxidation in mouse skeletal muscle ${ }^{31)}$, and reduce the body weight of high-fat diet-fed mice through an increase in ATGL level ${ }^{32)}$.

This study has certain limitations. First, MCFAs include caprylic acid, capric acid and lauric acid. We examined the beneficial effects of capric acid on mouse cardiac functions; however, other MCFAs such as caprylic acid and lauric acid may have similar effects that are not examined in the study. Second, the detailed molecular mechanisms of the capric acid-mediated improvement in LCFA metabolism were not clarified in this study.

\section{Conclusion}

In summary, the tricaprin $(+)$ diet improved the myocar- 
A

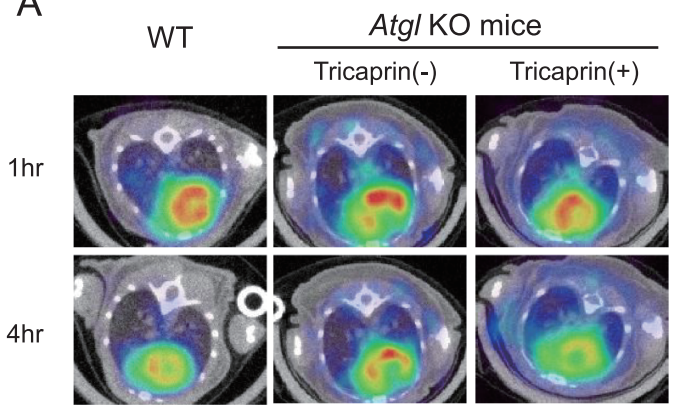

B

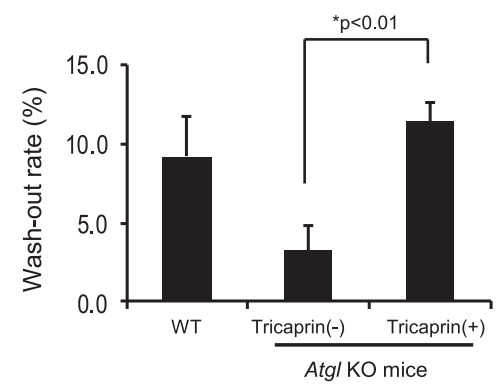

Fig. 1 Tricaprin diet improved myocardial LCFA metabolism.

(A) Representative images of WT mice that were fed tricaprin ( - ) diet and Atgl KO mice that were fed tricaprin $(-$ ) or tricaprin $(+) \operatorname{diet}(\mathrm{n}=4$ for each), obtained 1 and $4 \mathrm{~h}$ after BMIPP injection. (B) Bar graphs show the WOR in each group.
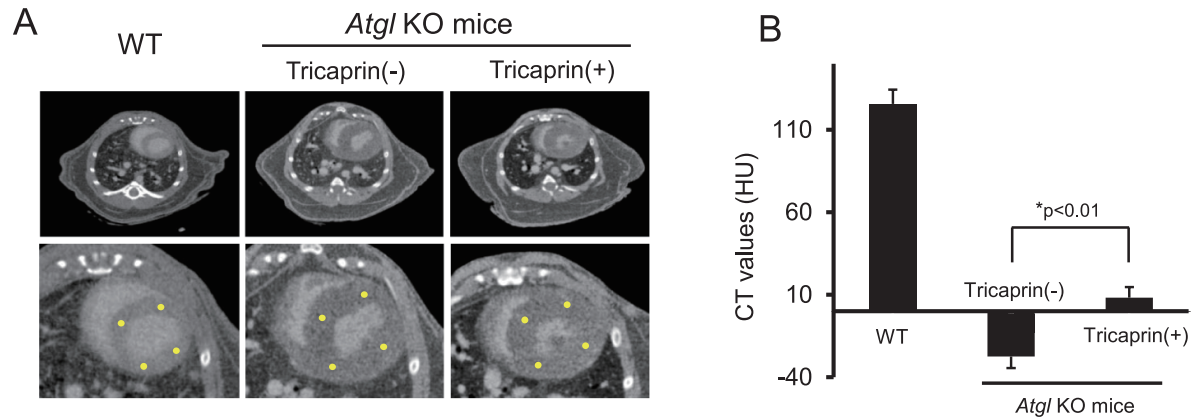

C
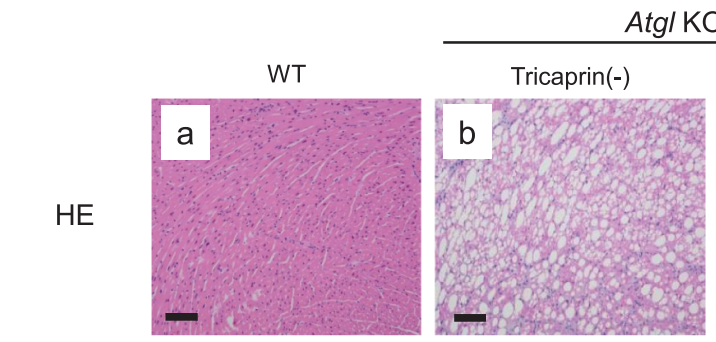

Atgl KO mice
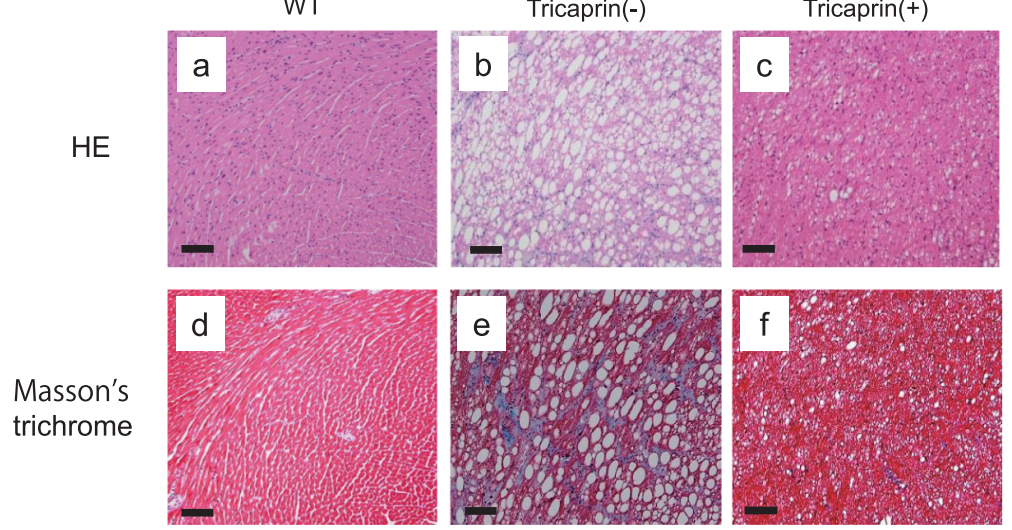

Fig. 2 Tricaprin diet reduced lipid deposition in Atgl KO mice.

(A) Representative images of micro-CT are shown. The upper panels are axial images of the whole body. The lower panels show enlarged images wherein yellow dots denote the ROIs for the measurement of CT values in the myocardium.

(B) The bar graphs show the mean CT values in each group ( $n=5$ for each group). Decreased CT values in the tricaprin $(-)$ diet-fed group improved after feeding the mice tricaprin $(+)$ diet. $\left({ }^{*} p<0.01\right)$.

(C) Representative images of HE staining in the hearts of WT mice that were fed tricaprin $(-) \operatorname{diet}(\mathrm{a})$, and KO mice that were fed tricaprin $(-) \operatorname{diet}(\mathrm{b})$ or tricaprin $(+) \operatorname{diet}(\mathrm{c})$. Masson's trichrome staining is also shown in $(\mathrm{d}-\mathrm{f})$. More lipid accumulation and fibrotic changes are observed in KO mice that were fed tricaprin $(-) \operatorname{diet}(\mathrm{b}$ and e). However, these are attenuated in KO mice that were fed tricaprin $(+) \operatorname{diet}(\mathrm{c}$ and f). Scale bar $=100 \mu \mathrm{m}$.

dial metabolism of LCFAs, reduced myocardial lipid deposition(Fig. 4), and improved left ventricular function in a mouse model of TGCV. We believe that the present data are sufficient to translate the use of tricaprin into clinical trials as nutritional therapeutic agents for patients with severe and critical symptoms of TGCV. 


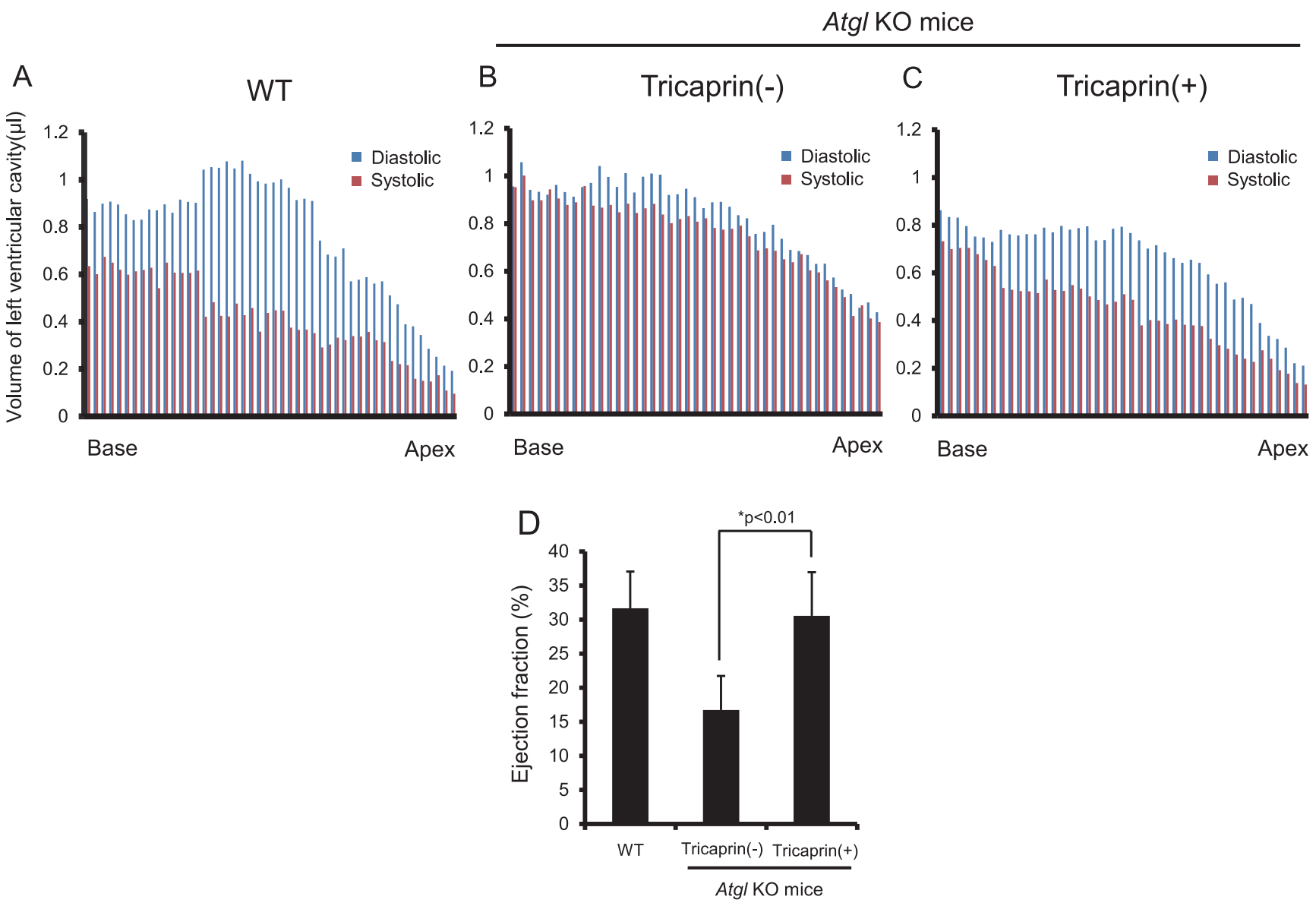

Fig. 3 Tricaprin diet improved cardiac dysfunction in Atgl KO mice

(A, B , C) Representative bar graphs indicate the volumes in each LV section from the base to the apex of the heart. Red and blue bars indicate segmental LV volumes at end-systole and end-diastole, respectively, in A(WT mice that were fed tricaprin $(-)$ diet $), \mathrm{B}(\mathrm{KO}$ mice that were fed tricaprin $(-)$ diet $)$, and $\mathrm{C}(\mathrm{KO}$ mice that were fed tricaprin $(+)$ diet). (D) The mean value of LVEF in KO mice that were fed tricaprin $(+)$ diet was significantly higher than that in mice fed tricaprin $(-) \operatorname{diet}(p<0.01)(\mathrm{n}=7$ for each group).
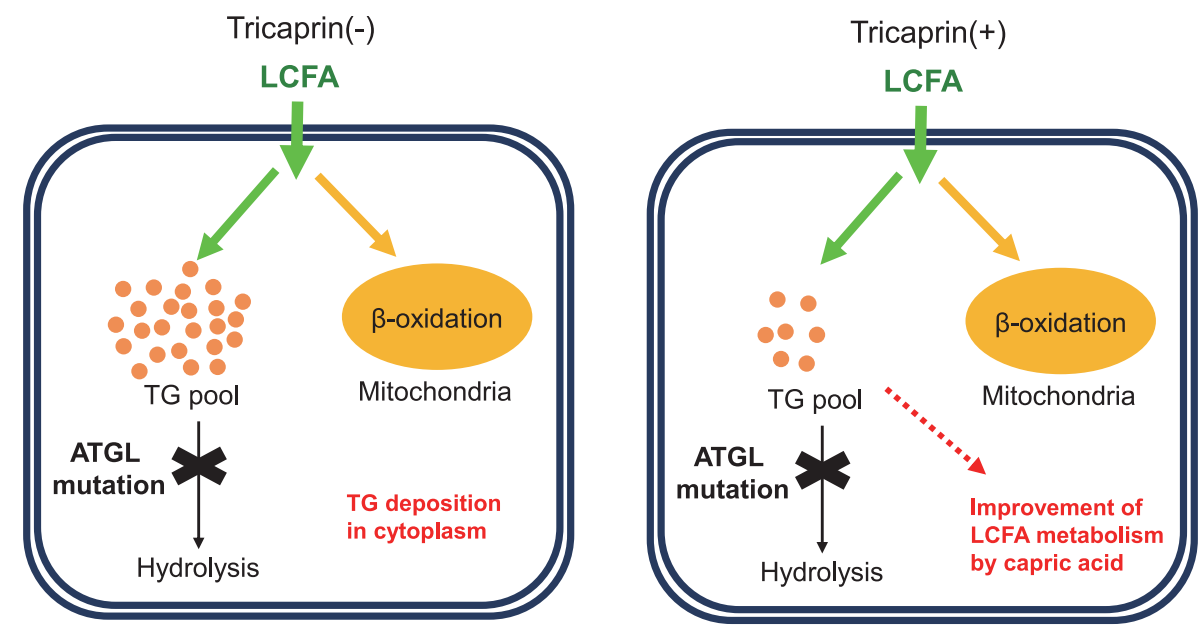

Fig. 4 Scheme of tricaplin regulated LCFA metabolism. 


\section{Acknowledgments}

The authors thank Professor Rudolf Zechner(University of Graz, Austria) for providing Atgl knockout mice. This study was partially supported by research grants for rare and intractable diseases from the Japan Agency of Medical Research and Development (AMED) (Grant No. 17ek0109092h0003) and from the Ministry of Health, Labour, and Welfare of Japan.

\section{Conflict of interests}

Ken-ichi Hirano received grants from Nihon Medi-Physics Co., Ltd., during the conduct of the study. Other authors declare that they have no conflicts of interest.

\section{References}

1) Hirano, K.; Ikeda, Y.; Zaima, N.; Sakata, Y.; Matsumiya, G. Triglyceride deposit cardiomyovasculopathy. $N$. Engl. J. Med. 359, 2396-2398(2008).

2) Hirano, K.; Ikeda, Y.; Sugimura, K.; Sakata, Y. Cardiomyocyte steatosis and defective washout of iodine123-beta-methyl iodophenyl-pentadecanoic acid in genetic deficiency of adipose triglyceride lipase. Eur. Heart J. 36, 580 (2015).

3) Ikeda, Y.; Hirano, K.; Fukushima, N.; Sawa, Y. A novel type of human spontaneous coronary atherosclerosis with triglyceride deposition. Eur. Heart J. 35, 875 (2014).

4) Zimmermann, R.; Strauss, J.G.; Haemmerle, G.; Schoiswohl, G.; Birner-Gruenberger, R.; Riederer, M.; Lass, A.; Neuberger, G.; Eisenhaber, F.; Hermetter, A.; Zechner, R. Fat mobilization in adipose tissue is promoted by adipose triglyceride lipase. Science 306, 1383-1386 (2004).

5) Haemmerle, G.; Lass, A.; Zimmermann, R.; Gorkiewicz, G.; Meyer, C.; Rozman, J.; Heldmaier, G.; Maier, R.; Theussl, C.; Eder, S.; Kratky, D.; Wagner, E.F.; Klingenspor, M.; Hoefler, G.; Zechner, R. Defective lipolysis and altered energy metabolism in mice lacking adipose triglyceride lipase. Science 312, 734-737 (2006).

6) Higashi, M.; Ikeda, Y.; Miyauchi, H.; Zaima, N.; Suzuki, A.; Li, M.; Kobayashi, K.; Naito, H.; Hirano, K. Imaging modalities for triglyceride deposit cardiomyovasculopathy. Ann. Nucl. Cardiol. 3, 94-102(2017).

7) Takagi, A.; Ikeda, Y.; Kobayashi, K.; Kobayashi, K.; Ikeda, Y.; Kozawa, J.; Miyauchi, H.; Li, M.; Hashimoto, C.; Hara, Y.; Yamaguchi, S.; Suzuki, A.; Toda, T.; Nagasaka, H.; Hirano, K.I. Newly developed selective immunoinactivation assay revealed reduction in adipose triglyceride lipase activity in peripheral leucocytes from patients with idiopathic triglyceride deposit cardiomyovasculopathy. Biochem. Biophys. Res. Commun. 495, 646-651 (2018).

8) Kaneko, K.; Kuroda, H.; Izumi, R.; Tateyama, M.; Kato, M.; Sugimura, K.; Sakata, Y.; Ikeda, Y.; Hirano, K.; Aoki, M. A novel mutation in PNPLA2 causes neutral lipid storage disease with myopathy and triglyceride deposit cardiomyovasculopathy: a case report and literature review. Neuromuscul. Disord. 24, 634-641 (2014).

9) Higashi, M.; Hirano, K.; Kobayashi, K.; Ikeda, Y.; Issiki, A.; Otsuka, T.; Suzuki, A.; Yamaguchi, S.; Zaima, N.; Hamada, S.; Hanada, H.; Suzuki, C.; Nakamura, H.; Nagasaka, H.; Miyata, T.; Miyamoto, Y.; Kobayashi, K.; Naito, H.; Toda, T. Distinct cardiac phenotype between two homozygotes born in a village with accumulation of a genetic deficiency of adipose triglyceride lipase. Int. J. Cardiol. 192, 30-32 (2015).

10) Hirano, K.; Tanaka, T.; Ikeda, Y.; Yamaguchi, S.; Zaima, N.; Kobayashi, K.; Suzuki, A.; Sakata, Y.; Sakata, Y.; Kobayashi, K.; Toda, T.; Fukushima, N.; Ishibashi-Ueda, H.; Tavian, D.; Nagasaka, H.; Hui, S.P.; Chiba, H.; Sawa, Y.; Hori, M. Genetic mutations in adipose triglyceride lipase and myocardial up-regulation of peroxisome proliferated activated receptor-gamma in patients with triglyceride deposit cardiomyovasculopathy. Biochem. Biophys. Res. Commun. 443, 574-579 (2014).

11) Hara, Y.; Kawasaki, N.; Hirano, K.; Hashimoto, Y.; Adachi, J.; Watanabe, S.; Tomonaga, T. Quantitative proteomic analysis of cultured skin fibroblast cells derived from patients with triglyceride deposit cardiomyovasculopathy. Orphanet. J. Rare Dis. 8, 197 (2013).

12) Hashim, S.A.; Arteaga, A.; Van Itallie, T.B. Effect of a saturated medium-chain triglyceride on serum-lipids in man. Lancet 1, 1105-1108 (1960).

13) Greenberger, N.J.; Skillman, T.G. Medium-chain triglycerides. N. Engl. J. Med. 280, 1045-1058(1969).

14) Augustin, K.; Khabbush, A.; Williams, S.; Eaton, S.; Orford, M.; Cross, J.H.; Heales, S.J. R.; Walker, M.C.; Williams, R.S.B. Mechanisms of action for the mediumchain triglyceride ketogenic diet in neurological and metabolic disorders. Lancet Neurol. 17, 84-93 (2018).

15) Labarthe, F.; Gelinas, R.; Des Rosiers, C. Mediumchain fatty acids as metabolic therapy in cardiac disease. Cardiovasc. Drugs Ther. 22, 97-106 (2008).

16) Nagasaka, H.; Hirano, K.; Ohtake, A.; Miida, T.; Takatani, T.; Murayama, K.; Yorifuji, T.; Kobayashi, K.; Kanazawa, M.; Ogawa, A.; Takayanagi, M. Improvements of hypertriglyceridemia and hyperlacticemia in Japanese children with glycogen storage disease type Ia by medium-chain triglyceride milk. Eur. J. Pediatr. 166, 1009-1016 (2007).

17) Hilaire, N.; Salvayre, R.; Thiers, J.C.; Bonnafe, M.J.; Negre-Salvayre, A. The turnover of cytoplasmic triacylglycerols in human fibroblasts involves two separate acyl chain length-dependent degradation pathways. $J$. 
Biol. Chem. 270, 27027-27034(1995).

18) Shinohara, H.; Shimada, H.; Noguchi, O.; Kubota, F.; Aoyama, T. Effect of medium-chain fatty acids-containing dietary oil on hepatic fatty acid oxidation enzyme activity in rats. J. Oleo Sci. 51, 621-626 (2002).

19) Tsuji, H.; Kasai, M.; Takeuchi, H.; Nakamura, M.; Okazaki, M.; Kondo, K.; Dietary medium-chain triacylglycerols suppress accumulation of body fat in a doubleblind, controlled trial in healthy men and women. $J$. Nutr. 131, 2853-2859 (2001).

20) Ogawa, A.; Nosaka, N.; Kasai, M.; Aoyama, T.; Okazaki, M.; Igarashi, O.; Kondo, K. Dietary medium- and longchain triacylglycerols accelerate diet-induced thermogenesis in humans. J. Oleo Sci. 56, 283-287 (2007).

21) Shrestha, R.; Hirano, K.I.; Suzuki, A.; Yamaguchi, S.; Miura, Y.; Chen, Y.F.; Mizuta, M.; Chiba, H.; Hui, S.P. Change in plasma total, esterified and non-esterified capric acid concentrations during a short-term oral administration of synthetic tricaprin in dogs. Anal. Sci. 33, 1297-1303 (2017).

22) Goodman, M.M.; Kirsch, G.; Knapp, F.F.Jr. Synthesis and evaluation of radioiodinated terminal p-iodophenyl-substituted alpha- and beta-methyl-branched fatty acids. J. Med. Chem. 27, 390-397 (1984) .

23) Kurata, C.; Tawarahara, K.; Taguchi, T.; Aoshima, S.; Kobayashi, A.; Yamazaki, N.; Kawai, H.; Kaneko, M. Myocardial emission computed tomography with iodine-123-labeled beta-methyl-branched fatty acid in patients with hypertrophic cardiomyopathy. J. Nucl. Med. 33, 6-13 (1992).

24) Oshima, M.; Higashi, S.; Kikuchi, Y.; Shirai, T.; Yokokawa, T.; Kaminaga, T.; Yasukochi, H.; Furui, S. Myocardial fatty acid metabolism in diabetic mice with 125IBMIPP. Ann. Nucl. Med. 12, 133-137 (1998).

25) Matsushita, S.; Naito, M.; Amano, A. Micro-computed tomography provides accurate measurement for cardiac function in infarcted rat heart. Open Journal of Medical Imaging 4, 72-79(2014).
26) Kim, A.J.; Francis, R.; Liu, X.; Devine, W.A.; Ramirez, R.; Anderton, S.J.; Wong, L.Y.; Faruque, F.; Gabriel, G.C.; Chung, W.; Leatherbury, L.; Tobita, K.; Lo, C.W. Microcomputed tomography provides high accuracy congenital heart disease diagnosis in neonatal and fetal mice. Circ. Cardiovasc. Imaging. 6, 551-559 (2013).

27) Yamamichi, Y.; Kusuoka, H.; Morishita, K.; Shirakami, Y.; Kurami, M.; Okano, K.; Itoh, O.; Nishimura, T. Metabolism of iodine-123-BMIPP in perfused rat hearts. $J$. Nucl. Med. 36, 1043-1050 (1995).

28) Brown-Harrison, M.C.; Nada, M.A.; Sprecher, H.; Vianey-Saban, C.; Farquhar, J.Jr.; Gilladoga, A.C.; Roe, C.R. Very long chain acyl-CoA dehydrogenase deficiency: Successful treatment of acute cardiomyopathy. Biochem. Mol. Med. 58, 59-65(1996).

29) Footitt, E.J.; Stafford, J.; Dixon, M.; Burch, M.; Jakobs, C.; Salomons, G.S.; Cleary, M.A. Use of a long-chain triglyceride-restricted/medium-chain triglyceride-supplemented diet in a case of malonyl-CoA decarboxylase deficiency with cardiomyopathy. J. Inherit. Metab. Dis. 33 Suppl 3, S253-256 (2010).

30) Hoy, A.J.; Bruce, C.R.; Turpin, S.M.; Morris, A.J.; Febbraio, M.A.; Watt, M.J. Adipose triglyceride lipase-null mice are resistant to high-fat diet-induced insulin resistance despite reduced energy expenditure and ectopic lipid accumulation. Endocrinology 152, 48-58 (2011).

31) Abe, T.; Hirasaka, K.; Kohno, S.; Tomida, C.; Haruna, M.; Uchida, T.; Ohno, A.; Oarada, M.; Teshima-Kondo, S.; Okumura, Y.; Choi, I.; Aoyama, T.; Terao, J.; Nikawa, T. Capric acid up-regulates UCP3 expression without PDK4 induction in mouse C2C12 myotubes. J. Nutr. Sci. Vitaminol. (Tokyo)62, 32-39 (2016).

32) Liu, Y.-h.; Xu, Q.; Zhang, X-s.; Wang, J.; Yu, X.-m; Yang, X.-y.; Xue, C.-y. Capric acid reduces body weight in C57BL/6J mice fed a high fat diet. Acta Nutr. Sin. 36, $5(2014)$. 